\title{
Informational Reinterpretation of the Mechanics Notions and Laws
}

\author{
Edward Bormashenko \\ Chemical Engineering Department, Engineering Faculty, Ariel University, P.O.B. 3, \\ 407000 Ariel, Israel
}

\begin{abstract}
The informational re-interpretation of the basic laws of the mechanics exploiting the Landauer principle is suggested. When a physical body is in rest or it moves rectilinearly with the constant speed, zero information is transferred; thus, the informational affinity of the rest state and the rectilinear motion with a constant speed is established. The analysis of the minimal Szilard thermal engine as seen from the noninertial frame of references is carried out. The Szilard single-particle minimal thermal engine undergoes the isobaric expansion relatively to the accelerated frame of references, enabling the erasure of 1 bit of information. The energy $\Delta Q$ spent by the inertial force for the erasure of 1 bit of information is estimated as: $\Delta Q \cong \frac{5}{3} k_{B} \bar{T}$, which is larger than the Landauer bound but qualitatively close to it. The informational interpretation of the equivalence principle is proposed: the informational content of the inertial and gravitational masses is the same.
\end{abstract}

Keywords: Landauer principle; inertial frame of reference; non-inertial frame of references; minimal thermal engine; equivalence principle.

\section{Introduction}

The Landauer Principle, suggesting the thermodynamic equivalent of information, attracted an essential interest of researchers in the last decade [1-5]. In its strictest, tightest, and simplest meaning, the Landauer principle states that the erasure of one bit of information requires a minimum energy cost equal to $k_{B} T \ln 2$, where $T$ is the temperature of a thermal reservoir used in the process and $k_{B}$ is Boltzmann's constant [6-7]. Landauer also applied the suggested principle to the transmission of information and re-shaped it as follows: an amount of energy equal to $k_{B} T \ln 2$ (where $k_{B} T$ is the thermal noise per unit bandwidth) is needed to transmit a bit of information, and more if quantized channels are used with photon energies $h v>k_{B} T$ [8]. Actually the Landauer principle converted the information into physical value, Rolph Landauer himself stated that the "information is physical" [7]. 
The precise meaning, evaluation and the interpretation of the Landauer principle were subjected to the intensive scientific discussion [9-10]. The author suggests that the Landauer Principle supports the physical paradigm proposed by J. A. Wheeler, which may be very briefly summarized as follows: "all things physical are informationtheoretic in origin", aphoristically reduced to "it from bit" [11]. Indeed, the Landauer principles enables the estimation of the mass of one bit of information [12] and to establish the mass-energy-information equivalence [4, 13]. The concept of information has been already successfully exploited for the grounding and clarification of the thermodynamics [14-18]. The present paper suggests the re-interpretation of the mechanics based on the informational approach. The information-based re-considering of the random molecular motion was undertook in ref. 19.

\section{Results and discussion}

\subsection{Informational re-interpretation of the first Newton Law}

Consider the first Newton Law in its traditional wording: "In an inertial frame of reference, an object either remains at rest or continues to move at a constant velocity, unless acted upon by a force" [20]. The First Newton Law was deeply criticized in the modern era of physics [20]. It was asserted, that actually the first Newton Law is not an empirical physical law, but the definition of the inertial frame of reference [20]. Whatever is the precise meaning of the first Newton Law it establishes the deep physical affinity of the rest and the rectilinear motion with the constant speed. Consider this affinity from the informational point of view. When a particle is in rest or it moves rectilinearly with the constant speed, no information is transferred from one object to another. Indeed, for transferring of at least one bit of the information from a transmitter to a receiver, the particle (it may be photon, electron or a macroscopic particle) should be emitted and absorbed. Both of these processes necessarily demand the acceleration (deceleration) of a particle [21]. When a particle is in rest or it moves rectilinearly with the constant speed, zero information is transferred. The informational re-interpretation clarifies the affinity of the rest and the rectilinear motion with the constant speed. Moreover, the informational re-definition of the inertial frame of reference becomes possible: the frame of reference is regarded as inertial when a free body located in this system does not exchange information with other physical objects.

\subsection{The Landauer principle and non-inertial frames of reference}

Now consider the motion of a particle as it seen from the non-inertial frame of reference from the informational point of view. Consider the computing device, 
exemplified by the single-particle (minimal) thermal engine, suggested by Leo Szilard in 1929 [22-24]. This minimal thermal engine is based on a particle $m$ enclosed within a chamber (cylinder) divided by half by a partition (piston) $M$, as shown in Figure 1. Finding of the particle $m$ in the certain (left or right) half of the chamber corresponds to the recording of 1 bit of information. When the partition is removed, the location of particle is uncertain, and this corresponds to the erasure of 1 bit of information. Location of a particle on the certain half of the chamber corresponds to "1", and the uncertain location of the particle corresponds to " 0 ", thus the single particle based computer provides the binary logical system [25]. The thermodynamic analysis of this computer-engine immediately gives rise to the Landauer bound, namely, the minimal energy necessary for isothermal erasing of one bit of information, which equals $k_{B} T \ln 2$ [23-25].

Consider the minimal thermal engine involving the particle $m$ and the partition $M$ which may slide in a frictionless way along the chamber, as shown in Figure 1. Address the action of the engine from the non-inertial frame of references moving with acceleration $\vec{a}$ relatively to engine, as shown in Figure 1. Both the particle $m$ and the partition $M$ will be exerted the inertial forces $\vec{F}_{i n, m}=-m \vec{a}$ and $\vec{F}_{i n, M}=-M \vec{a}$ [26]. As a result of the motion of the partition exposed to the inertial force $\vec{F}_{i n, M}$ the location of the particle will become uncertain, and it may be interpreted as erasure of 1 bit of information. The entire process may be seen as the isobaric expansion of the system under the inertial-force-inspired pressure: $P=\frac{\left|\vec{F}_{i n, M}\right|}{S}$, where $S$ is the cross-section of the partition. The energy $\Delta Q$ spent by the inertial force for the isobaric expansion (and erasure of 1 bit of information) is estimated as:

$$
\Delta Q=\Delta \varepsilon+P \Delta V \cong \frac{3}{2} k_{B} \Delta T+P \Delta V \cong c_{P} \Delta T \cong \frac{5}{2} k_{B} \Delta T=\frac{5}{2} k_{B} T_{1}=\frac{5}{3} k_{B} \bar{T},
$$

where $\Delta \varepsilon$ is the change in the kinetic energy of the particle, $\Delta V=V_{2}-V_{1}=2 V_{1}-$ $V_{1}=V_{1}$ is the change in the volume, occupied by the particle and $\Delta T=T_{2}-T_{1}=$ $2 T_{1}-T_{1}=T_{1}$ is the change of its temperature (i.e. an averaged kinetic energy) due the action of the inertial force $\vec{F}_{i n, m}$, and $\bar{T}=\frac{T_{1}+T_{2}}{2}=\frac{3}{2} T_{1}$ is the averaged across the isobaric expansion temperature. Eq. 1 implies that the motion of the particle is "thermalized" (i.e. randomized), and the notion of the temperature may be introduced be for the single-particle system. The conditions of "thermalizing" of small thermodynamic engines are discussed in detail in ref. 24. Eq. 1 actually returns us to 
within the numerical coefficient to the Landauer bound (consider that the Landauer bound defines the minimal energy necessary for the erasure of 1 bit of information). Of course, the rough estimation supplied by Eq. 1 should be taken cum grano salis. The use and correct understanding of this estimation is subtle; indeed the temperature is usually understood as the quantitative measure of the kinetic energy of the random motion of particles [27-28], whereas the inertial force gives rise to the directional motion of a particle. Anyway, it is recognized that inertial forces may be used for the erasure of information within the minimal Szilard-like engine. Reversing the acceleration and correspondingly the inertial force will give rise to the compression of the particle, and recording of 1 bit of information.

\subsection{The informational re-interpretation of the equivalence principle}

The understanding of the equivalence principle of general relativity may be ambiguous. ${ }^{21,29} \mathrm{We}$ adhere to the very tight interpretation of the equivalence principle implying the equivalence of the gravitational and inertial mass $M_{g r}=M_{i n} \cdot{ }^{30}$ Consider inertial mass $M_{\text {in }}$ in thermal equilibrium with surrounding (i.e. a thermal bath) under the temperature of $T$. The informational content $I$ of the inertial mass $M_{\text {in }}$ may be calculated according to the Landauer principles and refs. 4, 12-13 according to Eq. 2 :

$$
I=\frac{M_{i n} c^{2}}{k_{B} T \ln 2}=\frac{M_{g r} c^{2}}{k_{B} T \ln 2}
$$

Eq. 2 supplies the informational reinterpretation of the equivalence principle, namely the maximal informational content of the inertial and gravitational masses is the same. It is plausible to assume that the maximal number of bits that may be recorded by a particle in the thermal bath $I$ is a relativistic invariant (as well as that the entropy is the relativistic invariant [31]). Thus, Equation (2) implies the relativistic temperature transformation suggested by Ott [32] (for the detailed discussion see refs. 13, 31).

\section{Conclusions}

John Archibald Wheeler proposed rethinking of basic physical notions and laws within the informational paradigm, aphoristically summarized as "it from bit" [11]. The informational re-interpretation of thermodynamics has been already reported [14-18]. The present paper introduces informational re-interpretation of the basic notions and laws of mechanics, namely: the first Newton law and the principle of equivalence. The informational affinity of the rest and the rectilinear motion with a constant speed 
clarifies the first Newton law. When a physical body is in rest or it moves rectilinearly with the constant speed, zero information is transferred to surrounding physical objects. The informational interpretation of the Einstein general relativity equivalence principle is proposed: the informational content of the inertial and gravitational masses in thermal equilibrium with the surrounding bodies is the same. The analysis of the action of the minimal Szilard single particle thermal engine as seen from the non-inertial frame of references is performed. The Szilard minimal thermal engine undergoes the isobaric expansion relatively to the accelerated frame of references, enabling the erasure of 1 bit of information. The energy $\Delta Q$ spent by the inertial force for the erasure of 1 bit of information is estimated as: $\Delta Q \cong \frac{5}{3} k_{B} \bar{T}$ in a qualitative proximity to the Landauer bound.

\section{Funding}

This research received no external funding.

\section{Acknowledgments}

The author is thankful to Yelena Bormashenko for her kind help in preparing this paper.

\section{Conflicts of Interest}

The author declares no conflict of interests.

\section{References}

1. Kucia, R. A. Landauer's Principle as a special case of Galois Connection. Entropy 2018, 20 (12), 971.

2. Bérut, A.; Arakelyan, A.; Petrosyan, A.; Ciliberto, S.; Dillenschneider, R.; Lutz, E. Experimental verification of Landauer's principle linking information and thermodynamics. Nature 2012, 483, 187-189.

3. Herrera, L. The Gibbs Paradox, the Landauer Principle and the irreversibility associated with tilted observers. Entropy 2017, 19, 110.

4. Vopson, M. M. The mass-energy-information equivalence principle. AIP Adv. 2019, 9, 095206. 
5. Diamantini, M. C.; Trugenberger, C. A. Generalized Landauer bound as a universal thermodynamic entropy in continuous phase transitions. Phys. Rev. E 2014, 89, 052138.

6. Landauer, R. Dissipation and heat generation in the computing process. IBM J. Res. Dev. 1961, 5, 183.

7. Landauer, R. Information is physical. Phys. Today 1991, 44, 23-29.

8. Landauer, R. Minimal energy requirements in communication. Science 1996, 272, 1914-1918.

9. Norton, J. D. Eaters of the lotus: Landauer's principle and the return of Maxwell's demon. Stud. Hist. Philos. Sci. B 2005, 36, 375-411.

10. Norton, J. D. Waiting for Landauer. Stud. Hist. Philos. Sci. B 2011, 42, 184 198.

11. Wheeler, J. A. Information, physics, quantum: the search for links, Proceedings of the 3rd International Symposium on Foundations of Quantum Mechanics in the Light of New Technology, Tokyo, 1989, 354-368.

12. Herrera, L. The mass of a bit of information and the Brillouin's Principle. Fluct. Noise Lett. 2014, 13, 14500.

13. Bormashenko, Ed. The Landauer Principle: Re-Formulation of the Second Thermodynamics Law or a step to Great Unification? Entropy 2019, 21(10), 918.

14. Ben Naim, A. Shannon's Measure of information and Boltzmann's H-Theorem. Entropy 2017, 19, 48.

15. Ben-Naim, A. Information Theory; World Scientific: Singapore, 2017.

16. Ben-Naim, A. A Farewell to Entropy: Statistical Thermodynamics Based on Information; World Scientific: Singapore, 2008.

17. Ben-Naim, A. Entropy, the Truth the Whole Truth and Nothing but the Truth; World Scientific: Singapore, 2016.

18. Ben-Naim, A. Entropy and information theory: uses and misuses. Entropy 2019, $21(12), 1170$.

19. Müller, J. G. Information contained in molecular motion. Entropy 2019, 21, 1052.

20. Earman, J.; Friedman, M. The Meaning and status of Newton's Law of inertia and the nature of gravitational forces. Philosophy of Science 1973, 40 (3). 
21. Landau, L.; Lifshitz, E. M. The Classical Theory of Fields. vol. 2 (4th ed.), Butterworth-Heinemann, Oxford, 1975.

22. Szilard, L. über die Entropieverminderung in einem thermodynamischen System bei Eingriffen intelligenter Wesen. Zeitschrift für Physik 1929, 53 (11-12), $840-856$.

23. Lutz, E.; Ciliberto, S. Information: From Maxwell's demon to Landauer's eraser. Physics Today 2015, 68 (9), 30-35.

24. Bormashenko, Ed.; Shkorbatov, A.; Gendeman O. The Carnot engine based on the small thermodynamic system: Its efficiency and the ergodic hypothesis. Am. J. Physics 2007, 75, 911-915.

25. Bormashenko, Ed. Generalization of the Landauer Principle for computing devices based on many-valued logic. Entropy 2019, 21(12), 1150.

26. Goldstein, H. Classical Mechanics, Addison-Wesley Publishing Co, Reading, USA, 1959.

27. Baierlein, R. Thermal Physics, Cambridge University Press, Cambridge, UK, 1999.

28. Landau, L. D. ; Lifshitz, E. M. Statistical Physics, 3rd Ed., Course of Theoretical Physics, Vol. 5, Elsevier, Oxford, UK, 2011.

29. Ohanian, H. What is the principle of equivalence? Am. J. Physics 1977, 45, 903.

30. Roll, P, G.; Krotkov, R.; Dicke, R. H. The equivalence of inertial and passive gravitational mass. Annals of Physics 1964, 26, 442-517.

31. Tolman, R.C. Relativity, Thermodynamics and Cosmology; Oxford University Press: Oxford, UK, 1934.

32. Ott, X. Lorenz-Transformation der Waerme and der Temperatur. Z. Phys. 1963, 175, 70-104. (In German). 


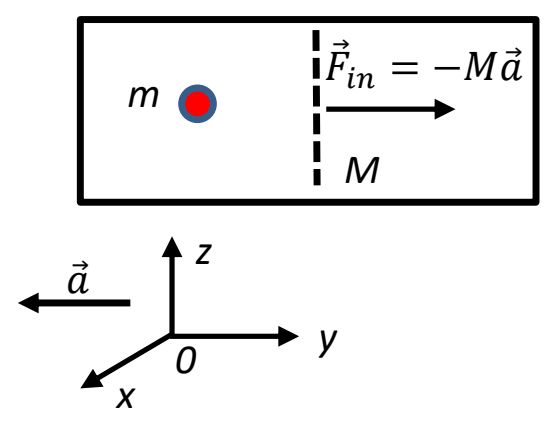

Figure 1. Finding of the particle $m$ in the certain (left or right) half of the chamber corresponds to the recording of 1 bit of information. The partition $\mathrm{M}$ is free to slide along the chamber. Frame of references $x y z$ moves with the acceleration $\vec{a}$ relatively to the chamber. The inertial forces $-m \vec{a}$ and $-M \vec{a}$ act on the particle $m$ and the partition $M$. The minimal thermal engine undergoes the isobaric expansion relatively to the frame of references $x y z$. 